\title{
A Survey on Delay Constraint Scheduling in Wireless Sensor Network
}

\author{
Pallavi P. Bambal ${ }^{1}$, Deepak C. Mehetre $^{2}$ \\ ${ }^{1}$ Department of Computer Engineering, K.J College of Engineering \& Management Research, Pune University, Pune, Maharashtra, India \\ ${ }^{2}$ Professor, Department of Computer Engineering, K.J College of Engineering \& Management Research, Pune University, Pune, \\ Maharashtra, India
}

\begin{abstract}
Wireless sensor network concept consist sensor nodes which are developed in the specific area senses and transmit data point to point. Delay constraint is the important point in the WSN. The delay guaranteed scheduling and flow control in WSN. Delay guaranteed are important for delay sensitive applications in WSN, Where data delivery is required in a timely manner e.g packet are send if they do not received a specified period or deadline. So we defined extension of the stability region, namely the constrained stability region in which the scheduling policy stabilizes the network regarding the delay constrained. The resulting policy that guarantees delay constraints.
\end{abstract}

Keywords: Scheduling, Wireless Sensor Network, Delay Scheduling, Sensor Nodes.

\section{Introduction}

A collection of nodes are organized into a cooperative network is called a Wireless sensor network. In WSN various types of small sensing and computation device are working in group to detect and monitor to change the environment in forests oceans and plains. WSN device have limited energy processing \&communication capabilities. Various WSN devices powered with small batteries are developed in remote area and it is easy to use of recharge and replace. Low energy algorithm and protocol are desing and development are essential for WSN network. WSN consist of multiple detection station is called as wireless sensor nodes. Each nodes is lightweight, small and portable. Few nodes are equipped with transducer, microcomputer, transceiver and power source. The transducer is generated electrical signals. The microcomputer processes and stores the sensor output. The transceiver is hard wired or wireless, it receive commands from central system and transmits data to that computer.[9]

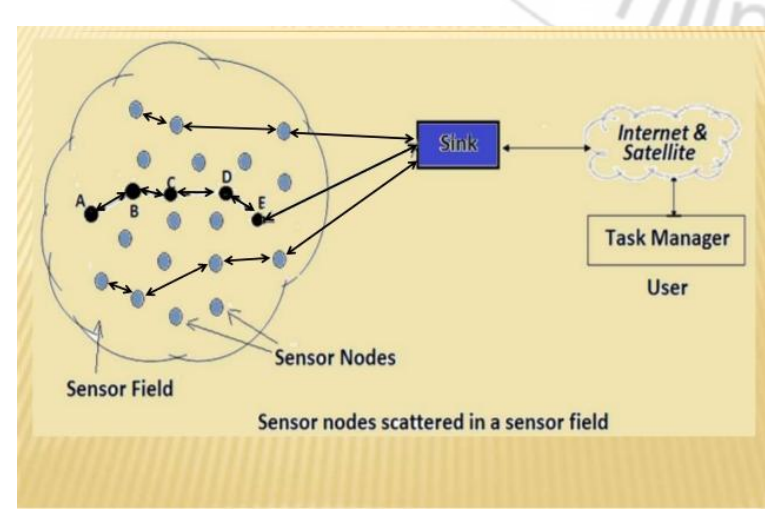

Figure 1: Block of Wireless Sensor network

\section{Scheduling}

The Processes manager are handles the removal running processes from the CPU and select another processes on the basis of particular strategy is called as processes scheduling. Processes Scheduling is the part of Multiprogramming. This
OS are allows more than one processes are loaded into executable memory at a time and those loaded process share the CPU using time multiplexing. Scheduling queue refere the process or devices. When process are enter the system then this process create a job queue. Devices queue are also maintain the OS. OS are Maintain all type of proceses such as a devices queue. Devices queue is a queue for which multiple procese are wating for a particular i/o devices each devices has own devices queue.[ book os]

\section{WSN Scheduling}

WSN scheduler is called packet Scheduler. Packet Scheduler manages the sequence of WSN packet in the transmit \& Receive queues of WSN controller. It is use circular Data buffer. There are various WSN Scheduler user for various types of OS. WSN define the large no. of Sensor nodes. Which are communicate in radio channel. Main Purpose of network is consist a physical variable. Gathering data and forwarding to the base station. Mesh topology are used in WSN.WSN communication can are either single hop or multihop depending upon the size of network. When combination of both type of network are used then WSN network are in certain way similar to MANET network (Mobile Adnoc Network). MANET devices are not as small as WSN device. The capacity of battery is high. WSN main purpose is energy consumption and all network processes have to be design with minimal energy demand [8]

\section{Classification for Carrier Techniques}

\subsection{CDMA (Code Division Multiple Access)}

Code division multiple access is a channel access method.It is used by various radio communication technologies. CDMA is example of multiple access where several transmitter can send information simultaneously over a single communication channel. This allows several users to share a band of frequency. CDMA is used as the access method in many mobile phone standards such as CDMA 


\section{International Journal of Science and Research (IJSR)}

ISSN (Online): 2319-7064

Index Copernicus Value (2013): 6.14 | Impact Factor (2015): 6.391

\subsection{FDMA: (Frequency Division Multiple Access)}

Frequency Division multiple Access is a channel access method. It is used in multiple access Protocols as a channelization Protocol. FDMA gives users an individual allocation of one or several frequency bands or channel. It is particularly common place in satellite communication. FDMA is distinct from frequency division duplexing while FDMA allows multiple users simultaneous access to a transmission system. FDMA also support demand assignment in addition to fixed assignment

\subsection{TDMA (Time Division Multiple Access)}

Time division multiple access is technology. It is used in digital cellular telephone communication that divides each cellular channel into three time slots in order to increases the amount of data that can be carrier. TDMA was first specified as a standard in EIA/TIA Interim Standard 54(IS 54). TDMA is also used for digital Enhanced cordless Telecommunications. TDMA is a type of time division multiplexing with the special point that instead of having one transmitter connected to one receiver. There are multiple transmitter in the case uplink from a mobile phone to a base station. This become particular difficult because the mobile phone can around and vary the timing advance required to make its transmission from its peers

\subsection{OFDMA (Orthogonal Frequency Division Multiple Access )}

OFDMA is a multiple user version of the popular orthogonal frequency division multiplexing digital modulation scheme. Multiple access is achieved in OFDMA by assigning subsets of subcarrier to individual user are allow simultaneous low data rate transmission from about the channel condition adaptive user to subcarrier assignment can be achieved. If the assignment is done sufficiently fast this further improves the OFDMA robustness to fast fading and narrow band channel interferences and make it possible to achieve even better system spectral Efficiency

\section{Literature Survey}

\subsection{Delay constrained optimal link scheduling in wireless sensor network}

Optimal link scheduling problem in wsn the optimal link scheduler are consider to assign time slot of different user to minimized the usage of data rate, delay bound and delay bound violation probability effective capacity are designed for mixed integer optimization problem. in this paper solved the optimal scheduler problem through the column generation based algorithm . medium access control (MAC) protocol are designed for optimal link strategy in WSN. Link scheduling problem are difficult to solve under signal-tointerference-plus-noise ratio (SINR) concept such type of scheduling problem are equivalent to the graph coloring problem[4]

4.2 Energy efficient Scheduling of Delay constraint: Result online and offline
In future wireless application is provided the high speed data link in limited bandwidth. Optimal offline scheduler packet arrivals before Scheduling. Online schedulers are compared to the offline scheduler in terms of delay and energy performance via analysis and simulation. Optimal energy efficient scheduling algorithm is minimizing the total transmission energy was develop for single transmission deadline and online are developed for large per packet delay. Firstly this paper analyzes for individual delay constraint model optimal packet transmission duration vector exhibit a symmetric property. This property makes simple and exact solution of average packet delay. Then we study two heuristic online scheduler for individual delay constraint model. First online scheduler generated the optimal static buffer flushing algorithm. And second online scheduler generated for Iterative minimum emptying time(IMET) algorithm[3]

\subsection{Energy Efficient TDMA Delay Scheduling:}

In this paper energy efficient TDMA protocols divided time into slots. Time Slots are defined for the proper communication in the network. This Schemes increases the delay $\&$ reduces the channel utilization significantly. TDMA link Scheduling minimize the number of time slot assigned while producing an interference free link scheduling \& shows NP complete problem . several algorithm are work on the this problem. if the TDMA link scheduling is used as mechanism in delay scheduling is used as mechanism in delay scheduling a node start the communication in numerous time with neighbors .If a sensor nodes start up to frequently .

The main contributions of this paper are summarized as follows:

(1) The scheduling problem in a new energy model, which is closer to realistic sensors.

(2) The contiguous link scheduling problem in WSNs, and prove it to be NP-complete.

(3)The centralized and distributed algorithms that have theoretical performance bound to the optimum of the problem.

(4)Develop simulations to show the efficiency of the proposed algorithms [10][11].

\subsection{Balanced-energy Delay Scheduling}

The Delay Technique has been used battery powered sensor for conserver the energy. Active \& inactive Sensor in the cluster which provided the redundant data. In this way they are manage the lifetime of network. Some research are suggest put redundant nodes into the network and allowing extra sensor to external the life time of network. When nodes goes to the seep state then it completely shut down only in wakeup mode they are leaving. In this paper use the probabilistic approach to balance the energy consumption for maintaining the energy consumption .This paper goal is that proposes and analyze the balanced energy scheduling (BS) scheme. [10]

\subsection{Optimal Delay Scheduling}




\section{International Journal of Science and Research (IJSR) \\ ISSN (Online): 2319-7064 \\ Index Copernicus Value (2013): 6.14 | Impact Factor (2015): 6.391}

A wireless sensor network whose packet delay periodically; however, rather than evaluating the system with a given delay control policy, we impose a cost structure and search for an optimal policy amongst a class of policies. In order to approach the problem in this manner, we need to consider a far simpler system than those used in the aforementioned studies. Thus, we consider only a single sensor node and focus on the tradeoffs between energy consumption and packet delay. As such, we do not consider other quality of service measures such as connectivity or coverage. The single node under consideration in our model has the option of turning its transmitter and receiver off for fixed durations of time in order to conserve energy. Doing so obviously results in additional packet delay. We attempt to identify the manner in which the optimal sleep schedule varies with the length of the sleep period, the statistics of arriving packets, and the charges assessed for packet delay and energy consumption.[12]

\subsection{Energy Efficient delay constraint scheduling}

This paper recently research effort on open vehicle routing problem researched are based on similar assumption \& constraint compared to sensor network therefore these techniques is provided valuable solution certain tricky problem in the wireless sensor network. So we develop the one collection protocol called EDAL(Energy efficient delay aware lifetime balancing data collection).

\subsection{Efficient Scheduling for Delay Constrained CDMA Wireless Sensor Networks:}

In this paper observed that the efficient scheduling and the resulting power allocation problem for a delay constrained CDMA WSN, which is modeled as a two-tiered network .Tired structure basically preferred in large-scale WSN. Multi- rate CDMA WSN facilitated by the multiple codes .each codes belonging to the virtual nodes. Scheduling problem seeing same in bin packing problem which is a NP complete. so we proposed scheduling strategy with polynomial complexity provides a near-optimal solution .result of this method is that saving the power with respect to TDMA

\subsection{Energy-efficient, Delay-aware, and Lifetime- balancing Data Collection Protocol}

This paper recently research effort on open vehicle routing problem researched are based on similar assumption \& constraint compared to sensor network therefore these techniques is provided valuable solution certain tricky problem in the wireless sensor network. So we develop the one collection protocol called EDAL(Energy efficient delay aware lifetime balancing data collection) .[1]

\section{Summary of Method Uses}

\begin{tabular}{|c|c|c|c|}
\hline Paper name & $\begin{array}{c}\text { Constraint } \\
\end{array}$ & Analytical Tools/Method & Achievement \\
\hline $\begin{array}{l}\text { 1. Delay constrained optimal link } \\
\text { scheduling in wireless sensor } \\
\text { network }\end{array}$ & $\begin{array}{l}\text { Data rate, delay bound and delay bound } \\
\text { violation probability. }\end{array}$ & $\begin{array}{l}\text { Medium access control protocol } \\
\text { it implement for optimal link } \\
\text { Scheduling. }\end{array}$ & $\begin{array}{l}\text { Column generation is } \\
\text { iterative algorithm for } \\
\text { solving the huge liner or } \\
\text { integer programming } \\
\text { problem }\end{array}$ \\
\hline $\begin{array}{c}\text { 2.Energy efficient Scheduling of } \\
\text { Delay constraint: Result online } \\
\text { and offline }\end{array}$ & $\begin{array}{c}\text { Packet size and unequal delay } \\
\text { constraint }\end{array}$ & $\begin{array}{l}\text { Energy efficient scheduling } \\
\text { algorithm. } \\
\text { Offline algorithm and two } \\
\text { heuristic online schedulers for } \\
\text { the individual delay constraint } \\
\text { model. }\end{array}$ & $\begin{array}{l}\text { Two algorithm such as } \\
\text { online or offline are } \\
\text { minimizing the transmission } \\
\text { energy and design the } \\
\text { individual delay constraint } \\
\text { model }\end{array}$ \\
\hline $\begin{array}{l}\text { 3. Balanced-energy delay } \\
\text { scheduling scheme for high- } \\
\text { density } \\
\text { cluster-based sensor networks }\end{array}$ & $\begin{array}{l}\text { The Distance-based Scheduling (DS) } \\
\text { The Randomized } \\
\text { Scheduling (RS) } \\
\text { The coefficient of variation of their } \\
\text { energy consumption. }\end{array}$ & $\begin{array}{l}\text { Analyze a Balanced-energy } \\
\text { Scheduling (BS) scheme. } \\
\text { Cluster-based sensor networks. }\end{array}$ & $\begin{array}{c}\text { Compared with the DS and } \\
\text { the RS schemes for sensor } \\
\text { clusters }\end{array}$ \\
\hline $\begin{array}{l}\text { 4.Eifficient Scheduling for delay } \\
\text { constraint CDMA }\end{array}$ & $\begin{array}{l}\text { Power allocation Problem for Delay } \\
\text { constraint CDMA WSN }\end{array}$ & $\begin{array}{l}\text { Two model in wsn } \\
\text { 1)intra cluster communication } \\
\text { 2)inter cluster communication }\end{array}$ & $\begin{array}{l}\text { This two model provided } \\
\text { near optimum solution with } \\
\text { sacrifice perform And } \\
\text { saving computational } \\
\text { complexity }\end{array}$ \\
\hline $\begin{array}{l}\text { 5.Energy-efficient, Delay-aware, } \\
\text { and Lifetime-balancing Data } \\
\text { Collection Protocol }\end{array}$ & $\begin{array}{c}\text { it has some limitation distributed } \\
\text { heuristic is design which is the best for } \\
\text { large scale networks. }\end{array}$ & $\begin{array}{l}\text { It is rebuilt from the existing } \\
\text { system called OVR which uses } \\
\text { NP-hard algorithm. }\end{array}$ & $\begin{array}{l}\text { EDAL is used to generate } \\
\text { routes that connect all } \\
\text { source nodes with shortest } \\
\text { path node and minimal total } \\
\text { path cost, under the } \\
\text { constraints of packet delay } \\
\text { requirements and load } \\
\text { balancing needs. }\end{array}$ \\
\hline $\begin{array}{l}\text { 6.Energy-Efficient Local Wake- } \\
\text { up Scheduling in } \\
\text { Wireless Sensor Networks }\end{array}$ & $\begin{array}{l}\text { Active sensor runs out of energy, } \\
\text { colony optimization based method. }\end{array}$ & $\begin{array}{c}\text { TDMA and MAC layer protocol. } \\
\text { Local wake-up } \\
\text { scheduling (LWS) strategy }\end{array}$ & $\begin{array}{l}\text { Improve the low data-rate } \\
\text { WSN. Simulation results } \\
\text { reveal that mc-ACO yields } \\
\text { better performance }\end{array}$ \\
\hline 7.Interference-Free Wakeup & All communication graphs. & Traditional wakeup scheduling, & The optimum number of \\
\hline
\end{tabular}




\section{International Journal of Science and Research (IJSR) \\ ISSN (Online): 2319-7064}

Index Copernicus Value (2013): 6.14 | Impact Factor (2015): 6.391

\begin{tabular}{|c|c|c|c|}
\hline $\begin{array}{c}\text { Scheduling with Consecutive } \\
\text { Constraints in Wireless Sensor } \\
\text { Networks }\end{array}$ & Grid topologies & compact wakeup scheduling & $\begin{array}{l}\text { time slots in a period for } \\
\text { trees and grid graphs }\end{array}$ \\
\hline $\begin{array}{l}\text { 8.Scheduler for multichannel } \\
\text { flow-level dynamics }\end{array}$ & $\begin{array}{l}\text { Full CSI Markov chain Single cell } \\
\text { (OFDM) }\end{array}$ & $\begin{array}{c}\text { Throughput-optimal } \\
\text { wireless systems with Not fully } \\
\text { backlogged Lagrangi an } \\
\text { multipliers } \\
\end{array}$ & $\begin{array}{l}\text { Multi-carrier } \\
\text { Stability }\end{array}$ \\
\hline $\begin{array}{l}\text { 9.Optimal scheduler for HSDPA } \\
\text { networks. }\end{array}$ & $\begin{array}{c}\text { Full CSI. } \\
\text { Not fully backlogged Error free } \\
\text { transmission } \\
\text { Traffic: Bernoulli }\end{array}$ & $\begin{array}{c}\text { Finite state markov chain } \\
\text { Dynamic programing markov } \\
\text { process. Single cell (HSDPA) } \\
\text { Downlink }\end{array}$ & $\begin{array}{l}\text { Decision process Fairness. } \\
\text { Error free transmission } \\
\text { Markov. }\end{array}$ \\
\hline $\begin{array}{l}\text { 10.A Survey on Scheduling } \\
\text { Algorithms for Wireless } \\
\text { Sensor Networks }\end{array}$ & $\begin{array}{l}\text { Wireless sensor networks (WSN). } \\
\text { Traffic analysis } \\
\text { Environmental monitoring Industrial } \\
\text { Process monitoring Tactical systems. }\end{array}$ & $\begin{array}{c}\text { Large-scale wireless } \\
\text { sensor networks. Time Division } \\
\text { Multiple Access Protocol. } \\
\text { MAC layer } \\
\end{array}$ & $\begin{array}{c}\text { Sensor networks. } \\
\text { Sensor network properties }\end{array}$ \\
\hline $\begin{array}{c}\text { 11.Lifetime Maximization in } \\
\text { Wireless Sensor Networks Using } \\
\text { Residual Energy Sleep } \\
\text { Scheduling }\end{array}$ & $\begin{array}{l}\text { Random deployment of sensors Delay } \\
\text { periods }\end{array}$ & $\begin{array}{c}\text { Residual energy Delay } \\
\text { scheduling (RESS) } \\
\text { Node self-scheduling scheme } \\
\end{array}$ & $\begin{array}{l}\text { Avoid redundancy. } \\
\text { Minimize energy } \\
\text { consumption } \\
\end{array}$ \\
\hline $\begin{array}{c}\text { 12.Energy-Efficient Sleep } \\
\text { Scheduling for Data Collection } \\
\text { and Aggregation }\end{array}$ & $\begin{array}{l}\text { Systematic gather. } \\
\text { Key challenge. }\end{array}$ & $\begin{array}{l}\text { MAC layer protocol } \\
\text { low-data-rate WSNs }\end{array}$ & $\begin{array}{l}\text { Consume energy for state } \\
\text { transition Energy } \\
\text { consumption }\end{array}$ \\
\hline
\end{tabular}

\section{Conclusion}

We have studied the scheduling with different type of wireless sensor network scheduling. We have focus on delay constraint scheduling in WSN schemes. There are various approaches for scheduling like Energy Efficient TDMA Delay Scheduling, Delay constrained optimal link scheduling, Balanced-energy Delay Scheduling efficient scheduling for delay constraint CDMA wireless sensor networks. Heterogeneous protocol is a type of protocol that is used in EDAL for over heading issues. We also introduce new rate adaption, packet loss techniques and improving the throughput, lifetime and energy balance techniques

\section{References}

[1] EDAL: an Energy-efficient, Delay-aware, and Lifetimebalancing Data Collection Protocolfor Heterogeneous Wireless Sensor NetworksYanjun Yao1, Qing Cao1, and Athanasios V. Vasilakos2, by 2015

[2] End-to-End Delay Constrained Routing and Scheduling for Wireless Sensor Networks Qing Wang*, Pingyi Fan*, Dapeng Oliver Wu $\mathrm{W}^{\dagger}$ and Khaled Ben Letaief by 2011

[3] Energy Efficient Scheduling with Individual Packet Delay Constraints: Offline and Online Results: Wanshi Chen Qualcomm Inc. San Diego, CA 92121Michael J. Neely and Urbashi Mitra by W Chen 2007

[4] Delay-Constrained Optimal Link Scheduling in Wireless Sensor Networks .Qing Wang, Dapeng $\mathrm{Wu}$ and Pingyi Fan, 2010

[5] Approaches for Combating Delay and Achieving Optimal Path Efficiency in Wireless Sensor Networks Agam Gupta1, Mansi Gupta2, Anand Nayyar3,2014

[6] Efficient Scheduling for Delay Constrained CDMA Wireless Sensor Networks Min Chen Changyoon Oh Aylin Yener 2006

[7] I.F. Akyildiz, W. Su, Y. Sankarasubramaniam, E. Cayirci,"Asurvey on wireless sensor networks", IEEE Common. Mag. 41 (8) (2003) 102-114.
[8] G.J. Potties, W.J. Kaiser, "Wireless integrated network sensors", Common. ACM 43 (5) (2001) 51-58.

[9] J.M. Reason, J.M. Rabies, "A study of energy consumption and reliability in a multi-hop sensor network", ACM Mobile Computer. Common. Rev. 8 (1) (2004)

[10] W. Ye, J. Heinemann, D. Estrin, “An energy-efficient MAC protocol for wireless sensor networks", in: Proceedings of the IEEE INFOCOM, vol. 3, 2003.

[11]W.B. Hein Zelman, A.P. Chandrakasan, H. BalaKrishnan, "application specific protocol architecture for wireless micro sensor networks", IEEE Trans. Wireless Common. 1 (4) (2002).

[12] U. Cetintemel, A. Flinders, Y. Sun, "Power-efficient data dissemination in wireless sensor networks", in: Proceedings of the ACMMobiDE'03, 2003.

[13] A. Manjeshwar, D. Agrawal, "TEEN: a routing protocol for enhanced efficiency in wireless sensor networks", in: Proceedings of the IEEE IPDPS 2001, pp. 20092012.

[14] S. Lindsey, C. Raghavendra, K.M. Siva lingam, "Data gathering algorithms in sensor networks using energy metrics"IEEE Trans. Parallel Diatribe. Syst. 13 (9) (2002). 